\title{
A bioinformatics potpourri
}

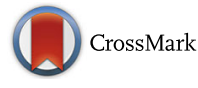

\author{
Christian Schönbach ${ }^{1 *}$, Jinyan $\mathrm{Li}^{2}$, Lan $\mathrm{Ma}^{3}$, Paul Horton ${ }^{4}$, Muhammad Farhan Sjaugi ${ }^{5}$ and Shoba Ranganathan ${ }^{6^{*}}$
}

From 16th International Conference on Bioinformatics (InCoB 2017)

Shenzhen, China. 20-22 September 2017

\begin{abstract}
The 16th International Conference on Bioinformatics (InCoB) was held at Tsinghua University, Shenzhen from September 20 to 22, 2017. The annual conference of the Asia-Pacific Bioinformatics Network featured six keynotes, two invited talks, a panel discussion on big data driven bioinformatics and precision medicine, and 66 oral presentations of accepted research articles or posters. Fifty-seven articles comprising a topic assortment of algorithms, biomolecular networks, cancer and disease informatics, drug-target interactions and drug efficacy, gene regulation and expression, imaging, immunoinformatics, metagenomics, next generation sequencing for genomics and transcriptomics, ontologies, post-translational modification, and structural bioinformatics are the subject of this editorial for the InCoB2017 supplement issues in BMC Genomics, BMC Bioinformatics, BMC Systems Biology and BMC Medical Genomics. New Delhi will be the location of InCoB2018, scheduled for September 26-28, 2018.
\end{abstract}

Keywords: InCoB, International conference on bioinformatics, APBioNet, Asia-Pacific bioinformatics network

\section{Introduction}

InCoB2017 was co-organized by The University of Technology Sydney, Tsinghua University, Graduate School at Shenzhen and APBioNet [1]. Six keynote and two invited talks covered the latest bioinformatics applications and big data developments in basic and applied biomedical research. The theme of big data-driven bioinformatics and precision medicine was highlighted in a panel discussion on its current status and future. Jianzhu Chen (Koch Institute for Integrative Cancer Research, Massachusetts Institute of Technology) presented bioinformatics-driven immunological research towards the identification of transcription factors in memory $\mathrm{CD}^{+} \mathrm{T}$ cell development, and the screening of bioactive and natural compounds that are able to induce human macrophages into either inflammatory or anti-inflammatory states. Yuelong Shu of the School of Public Health (Shenzhen) at Sun Yat-sen University and WHO Collaborating Center for Reference and Research on Influenza demonstrated how large-scale sequencing of human influenza virus in combination with antigenic surveillance of hemagglutinin using the computational

\footnotetext{
*Correspondence: chris@kumamoto-u.ac.jp; shoba.ranganathan@mq.edu.au ${ }^{1}$ International Research Center for Medical Sciences, Graduate School of Medical Sciences, Kumamoto University, Kumamoto 860-0811, Japan

${ }^{6}$ Department of Chemistry and Biomolecular Sciences, Macquarie University, Sydney, NSW 2109, Australia

Full list of author information is available at the end of the article
}

platform PREDAC improved vaccine strain recommendations for China.

Single cell RNA sequencing can reveal small differences among cells which are important to know in understanding of cellular responses to signals and variations among one cell type. Yong Hou (BGI-Shenzhen) gave in his invited talk a comprehensive overview of single cell sequencing, its application in cancer research and potential to improve cancer diagnosis. Limsoon Wong (National University of Singapore) provided insight into reproducibility and coverage issues of mass spectrometry-based proteomics data, and introduced algorithms that produce more robust and biologically meaningful proteomic profiling results.

Two keynotes covered epigenetic modifications in embryonic stem cells from the perspectives of miRNA regulation and networks of chromatin-related proteins. Xiujie Wang (Institute of Genetics and Developmental Biology, Chinese Academy of Sciences) reported on clusters of miRNAs that were positively correlated with the pluripotency level of embryonic stem cells. One of the miRNAs was involved in a new form of mRNA regulation through $\mathrm{N}^{6}$-methyladenosine modification. Alfonso Valencia (Barcelona Supercomputing Center) concentrated in his talk on network-based approaches in epigenomics, evolution and biomedicine on the role of 5-hydroxymethylcytosine as a communication hub in the chromatin network of embryonic stem cells, and concluded with a network property analysis that revealed 
Table 1 Summary of articles arranged by topic groups

\begin{tabular}{ll}
\hline Topic & Key finding or features \\
\hline Algorithms & Divisive hierarchical maximum likelihood clustering (DRAGON), reduced search complexity $\mathrm{O}\left(\mathrm{n}^{2} \mathrm{c}\right)$ [8]. \\
& 2D-EM two-step clustering approach applied to transcriptome and methylome data; filtering produces a feature \\
& matrix which is used as input for clustering by modified EM [9]. \\
& Deep learning; convolutional neural network (CNN) using gradient boosted feature selection for classification of \\
& B-lactamases [10]. \\
& Prediction of protein complexes from protein-protein interaction networks utilizing gene expression data and \\
& protein functional annotations; CPredictor3.0 [1 1]. \\
& Boolean network model simulation of signaling networks; changes of modularity and robustness by edge-removal \\
& mutations [12].
\end{tabular}

Integrated protein-protein interaction network construction using DIP, Biogrid, Reactome and HPRD data; refinement/correction using relationship of functional similarity and proximity scores [13].

Boolean network modeling; topology comparison of gene-gene dynamics influence and gene-gene molecular interaction networks [14].

Integration of gene regulatory network inference with constraint-based metabolic models to simulate growth phenotype and exchange fluxes [15].

Causal relationship detection between gene pairs for short time-series gene expression data (E. coli; yeast) based on lagged-coordinate delay embedding theorem [16].

Cell fate predictions derived from polynomial modeling of human pancreatic single-cell gene expression data [17].

Case study of network bi-stability and positive/negative feedback loops in TGF- $\beta 1$ activation [18].

Cancer and disease informatics
Drug-target interactions and drug efficacy

Gene expression and regulation
Multi-view clustering method with enhanced consensus; breast cancer sub-typing and survival analysis [19].

Network hubs as prognostic signatures in ovarian cancer, breast cancer and glioblastoma multiforme selected by Cox regression for correlating DNA methylation levels with outcome [20].

Analysis pipeline in Python to classify tumors using a supervised machine-learning algorithm that predicts mutation status based on transcriptional patterns [21].

Breast cancer outcome predictions from microarray data using Hadamard kernel [22].

Application of node-weighted Steiner tree approach to identify proteins and protein-protein interactions in PI3K/Akt and MAPK signaling pathways; subnetwork identification [23].

Tensor decomposition-based unsupervised feature extraction from gene expression data infers genes that induce post-traumatic stress disorder-mediated heart diseases and potential therapeutic targets [24].

Graph regression-based approach which creates a unified framework for predicting binary, discrete and continued IncRNA-disease associations [25].

Network consistency projection for human microbe-disease association predictions assuming that microbes with similar functions may have similar associated/not associated patterns with similar diseases [26].

Target control problem with objectives-guided optimization algorithm to identify drivers (e.g. drug target nodes or network biomarkers) controlling targets in disease-associated networks [27].

Ligand-based quantitative structure-activity relationship modeling using Random Forest for drug target identification; web server application [28].

Whole-body physiologically based pharmacokinetic modelling using constraint-based perturbation analysis with cluster Newton method; can handle mixed patient-dependent and patient-independent parameters [29].

Ternary status based integer linear programming approach for MCF7 breast cancer cell line specific pathway network reconstruction and prediction of treatment efficacy of compounds using prior knowledge of literature and phosphoproteomic data [30].

Core pharmacophore anchor model screening of FDA drugs to identify candidate dengue virus NS3 protease inhibitors [31].

Dependency-based deep neural network model for drug-drug interaction feature extraction form Drug Bank [32].

Prediction of human transcriptional target genes using open chromatin regions, ChIP-seq data and transcription factor binding sites [33].

Identification of phased secondary small interfering RNAs and miRNAs targeting PHAS loci in Panax notoginseng [34]. Identification candidate tissue-specific circRNAs using bi-clustering of RNA-Seq-derived expression profiles [35].

Multi-factor analysis of differential co-expression of breast cancer microarray data; identified differentially co-expressed sets containing ESR1 and CXCL13 [36]. 
Table 1 Summary of articles arranged by topic groups (Continued)

Topic
Imaging
Immuno-informatics
transcript-omics

transcript-omics

Ontologies

Post-translational modification sites

Structural bio-informatics Key finding or features

Random Forest approach that uses motif combinations in prediction of cell-type-specific transcription factor binding sites [37].

Discriminant filter bank approach for extracting EEG signal features using common spatial patterns; low misclassification rate [38].

MatQuantify is a software for assessment of fluorescence microscopy images of mitotic spindles and their architecture changes [39].

Phytoplankton and zooplankton image classification using a non-linear multiple kernel learning approach [40].

Computational methodology pipeline to process, predict and analyze potential T cell epitopes using influenza A, dengue, West Nile hepatitis A and HIV-1 virus sequence data [41].

NetCTL-bases predictions of HLA-A2, -A3 and -B7 supertype-restricted Zaire ebolavirus T cell epitope canidates [42].

Agent based-model to simulate citrus-derived flavones as vaccine adjuvants against human papilloma virus 16; mouse in vivo result confirmation [43].

Investigation of differences in cellular A-to-I RNA editing activities upon infection with influenza A virus H1N1 and H3N2 [44].

RNA-Seq based analysis of differential innate immune response of RNA-Seq human cells infected with H1N1,H3N1, H5N1, HALo mutant and H7N9 and chicken and quail cells infected with H5N1 and H5N2 [45].

Cellular RNA editing analysis of C. albicans infected human epithelial cell lines and mouse in vivo infected tongue and kidney tissues [46].

16sPIP analysis pipeline for classification of $16 \mathrm{~S}$ rDNA NGS data and screening of 346 clinically relevant pathogens [47].

CoMet binning workflow was used to assess contig coverage in combination with GC content for automated binning of a single and multi-strain metagenomic samples [48]

Metagenomic and -transcriptomic analysis of oolong teas to identify dominant microbial species and their anti-microbial peptides [49].

Computational pipeline ezTree infers marker genes and maximum likelihood phylogenetic trees from uncultivated prokaryotic genomes [50].

GTZ is a fast and lossless compression tool for cloud computing of FASTQ files; data transmission can overlap with compression; [51].

Coverage-dependent (from RNA sample concentration) RNA-Seq approach using a Bayesian method that infers the posterior distribution of a true gene count [52].

GT-WGS is a distributed whole-genome computing system based on Amazon Web Service cloud computing platform [53].

Pan-genome tool PGAP-X is a cross-platform software to analyze and visualize genome structure dynamics and and gene content [54].

Pará rubber (H. brasiliensis) genome transcriptome database [55].

InfAcrOnt tool can calculate similarities between terms across different ontologies and support the identifcation of novel relationships [56].

CroGO2 is an iterative ranking-based method that measures similarities of cross-categories GO terms using GO term level and interaction information in gene co-function networks [57]

Ontology of Chinese Medicine for Rheumatism represents 26 anti-rheumatism Chinese drugs together with their chemical ingredients, adverse effects and related information [58].

MDD-Carb is a tool for prediction of carbonylation sites utilizes maximal dependence decomposition and profile hidden Markov models [59].

SUCCESS is a SVM-based tool that predicts succinylation sites using structural and evolutionary information of amino acids [60].

CruxPTM is a platform for structure-based analysis post-translational modifications in context of PPI and drug binding [61].

Molecular dynamics analysis of charge states of balanol analogues that are ATP competitive inhibitors but nonselective for protein kinases $A$ and $C$ [62].

DeepSacon tool is a sparse autoencoder-based deep neural network to predict solvent accessibility and contact numbers [63].

R3D-BLAST2 is an improved search tool for similar RNA 3D substructures that can handle mmCIF files [64]. 
inverse as well as direct co-morbidities between Alzheimer's disease, glioblastoma and lung cancer.

Saman Halgamuge (The Australian National University) and Mindy Shi (University of North Carolina at Charlotte) offered in their presentations an impressive demonstration of deep learning applications. Saman Halgamuge showed in his keynote successful applications of unsupervised deep learning in the areas of direct drug-brain interactions, drug repositioning and multi-electrode array workflow applications for screening pharmacological compounds. Mindy Shi utilized deep learning to construct predictive models for quantitative trait locus network analysis using genomic and interactome data.

The Annual General Meeting of APBioNet on September 20th was opened with the President's Report. Among the reporting items was a new simplified membership fee structure with details available at APBioNet website [2], and plans to utilize BioRxiv preprint server [3] and its feature to transfer manuscripts to partnering journals for article submissions related to InCoB or InSyB (International Symposium of Bioinformatics). The winner of the bid for InCoB2018, Shandar Ahmad, introduced next year's conference venue at Jawaharlal Nehru University, New Delhi [4]. Jim Hogan (Queensland University of Technology) presented an Expression of Interest (EOI) to host InCoB2019 or InCoB2020 at Gold Coast, Australia. Parties interested in hosting InCoB or InSyB as stand-alone, joint or back-toback events are encouraged to submit an EOI through APBioNet's website [5].

\section{Manuscript submission and review}

In total 152 manuscripts were submitted through EasyChair conference management system [6] for consideration for publication as InCoB2017 supplement articles in Bioinformatics, BMC Genomics, BMC Bioinformatics, BMC Systems Biology, BMC Medical Genomics, IEEE/ ACM Transactions on Computational Biology and Bioinformatics (TCBB), Journal of Bioinformatics and Computational Biology (JBCB) or PeerJ. After peer review by at least two reviewers of the Program Committee comprising 121 members, supported by 27 external sub-reviewers (Additional file 1), 65 (42.7\%) manuscripts were provisionally accepted in revised form before the conference, pending final editorial approval. Fifty-seven articles are published in InCoB2017 supplement issues of BMC Bioinformatics (22) BMC Medical Genomics (7), BMC Systems Biology (14) and BMC Genomics (14). Eight articles will appear in PeerJ (1), JBCB (3), TCBB (3) and Bioinformatics (1). Best Paper Awards in the categories Gold, Silver and Bronze were given to authors of 28 manuscripts (Additional file 2). The articles included in the four BMC supplement issues are briefly summarized in Table 1 according to 12 topic groups arranged in alphabetical order.

\section{Conclusion}

The potpourri of bioinformatics research output showcased at InCoB2017 reflects APBioNet's goal to cater to a diverse range of practitioners and developers in the field. One of the highly cited articles of the InCoB conference series is an evaluation of human protein-protein interaction data in the public domain by Mathivanan et al. [7] with an average of ten citations per year. The paper was presented at InCoB2006 in New Delhi where next year's conference will be held.

\section{Additional files}

Additional file 1: List of InCoB2017 Reviewers. (PDF 52 kb)

Additional file 2: InCoB2017 Best Paper Awards. (PDF 73 kb)

\section{Acknowledgements}

We thank all reviewers and volunteering students and staff of Graduate School at Shenzen, Tsinghua University for their time and effort. We also thank Precision Medicine Research Center of Taihe Hospital (Hubei), School of Public Health (Shenzhen) at Sun Yat-sen University, School of Electrical and Information Engineering at Anhui University of Technology and International Society for Computational Biology for supporting InCoB2017.

\section{Funding}

The publication charge for this article was funded by APBioNet Ltd., Singapore. The funder had no role in the decision to publish or preparation of the manuscript.

Availability of data and materials Not applicable.

\section{About this supplement}

This article has been published as part of BMC Genomics Volume 19 Supplement 1, 2018: 16th International Conference on Bioinformatics (InCoB 2017): Genomics. The full contents of the supplement are available online at https://bmcgenomics.biomedcentral.com/articles/supplements/ volume-19-supplement-1.

\section{Authors' contributions}

CS and SR wrote manuscript. JL and LM organized the conference. CS, PH, MFS and SR managed reviewer assignments and communication with authors. All authors read and approved the final manuscript.

Ethics approval and consent to participate

Not applicable.

Consent for publication

Not applicable.

\section{Competing interests}

CS, PH and MFS are elected office bearers of APBioNet. SR is a member of the Board of Directors of APBioNet Ltd., Singapore. All other authors have declared that no competing interests exist.

\section{Publisher's Note}

Springer Nature remains neutral with regard to jurisdictional claims in published maps and institutional affiliations.

\section{Author details}

${ }^{1}$ International Research Center for Medical Sciences, Graduate School of Medical Sciences, Kumamoto University, Kumamoto 860-0811, Japan. ${ }^{2}$ The Advanced Analytics Institute, University of Technology Sydney, Sydney, NSW 2007, Australia. ${ }^{3}$ Graduate School at Shenzhen, Tsinghua University,

Shenzhen 518055, People's Republic of China. ${ }^{4}$ Artificial Intelligence Research 
Center, National Institute of Advanced Industrial Science and Technology, Tokyo 135-0064, Japan. ${ }^{5}$ School of Data Sciences, Perdana University, 43400 Serdang, Malaysia. ${ }^{6}$ Department of Chemistry and Biomolecular Sciences, Macquarie University, Sydney, NSW 2109, Australia.

\section{Published: 19 January 2018}

\section{References}

1. InCoB2017. https://incob.apbionet.org/. Accessed 14 Nov 2017.

2. APBioNet membership. https://www.apbionet.org/membership/. Accessed 14 Nov 2017.

3. BioRxiv. https://www.biorxiv.org/. Accessed 14 Nov 2017.

4. InCoB2018. http://www.incob2018.org/. Accessed 14 Nov 2017.

5. APBioNet EOI. https://www.apbionet.org/expression-of-interest-eoi-to-hostapbionet-conference-and-symposium/. Accessed 14 Nov 2017.

6. EasyChair. http://easychair.org/. Accessed 14 Nov 2017.

7. Mathivanan S, Periaswamy B, Gandhi TK, Kandasamy K, Suresh S, Mohmood R, Ramachandra YL, Pandey A. An evaluation of human protein-protein interaction data in the public domain. BMC Bioinformatics. 2006;7 Suppl 5:S19.

8. Sharma A, Lopez Y, Tsunoda T. Divisive hierarchical maximum likelihood clustering. BMC Bioinformatics. 2017;18(Suppl 16):S12.

9. Sharma A, Kamola PJ, Tsunoda T. 2D-EM clustering approach for highdimensional data through folding feature vectors. BMC Bioinformatics. 2017; 18 Suppl 16:S17.

10. White C, Ismail HD, Saigo H, Dukka BKC. CNN-BLPred: a convolutional neural network based predictor for $\beta$-lactamases (BL) and their classes. BMC Bioinformatics. 2017;18(Suppl 16):S19.

11. Xu Y, Zhou J, Zhou S, Guan J. CPredictor3.0: effectively detecting protein complexes from PPI networks with expression data and functional annotations. BMC Systems Biol. 2017:11(Suppl 7):S4.

12. Truong $C D$, Kwon Y-K. Investigation on changes of modularity and robustness by edge-removal mutations in signaling networks. BMC Systems Biol. 2017:11(Suppl 7):S5.

13. Guo M, Wang C, Liu X, Wang S. Refine gene functional similarity network based on interaction networks. BMC Bioinformatics. 2017;18(Suppl 16):S16.

14. Mazaya M, Trinh H-C, Kwon Y-K. Construction and analysis of gene-gene dynamics influence networks based on a Boolean model. BMC Systems Biol. 2017;11(Suppl 7):S9.

15. Banos DT, Elati M, Trébulle P. Integrating transcriptional activity in genomescale models of metabolism. BMC Systems Biol. 2017;11(Suppl 7):S7.

16. Zhang B-G, Li W, Shi Y, Liu X, Chen L. Detecting causality of short timeseries data by prediction of topologically equivalent attractors. BMC Systems Biol. 2017;11(Suppl 7):S12.

17. Ma L, Jie ZJ. A polynomial based model for cell fate prediction in human diseases. BMC Systems Biol. 2017;11(Suppl 7):S2.

18. Li H, Venkatraman L, Narmada BC, White J, Yu H, Tucker-Kellogg L. Computational analysis reveals the coupling between bistability and the sign of a feedback loop in a TGF-B1 activation model. BMC Systems Biol. 2017;11(Suppl 7):S8.

19. Cai M, Li L. Subtype identification from heterogeneous TCGA datasets on a genomic scale by multi-view clustering with enhanced consensus. BMC Med Genet. 2017;18(Suppl 4):S6.

20. Hu W, Zhou X. Identification of prognostic signature in cancer based on DNA methylation interaction network. BMC Med Genet. 2017;18(Suppl 4):S7.

21. Dayton J, Piccolo $\mathrm{S}$. Classifying cancer genome aberrations by their mutually exclusive effects on transcription. BMC Med Genet. 2017;10(Suppl 4):S3.

22. Jiang H, Ching W-K, Cheung W-S, Hou W, Yin H. Hadamard kernel SVM: applications for breast cancer outcome predictions. BMC Systems Biol. 2017; 11(Suppl 7):S14

23. Sun Y, Ma C, Halgamuge S. The node-weighted Steiner tree approach to identify elements of cancer-related signaling pathways. BMC Bioinformatics. 2017;18(Suppl 16):S5

24. Taguchi Y-H. Tensor decomposition-based unsupervised feature extraction identifies candidate genes that induce post-traumatic stress disordermediated heart diseases. BMC Med Genet. 2017;10(Suppl 4):S2.

25. Shi J-Y, Huang $\mathrm{H}$, Zhang $Y-N$, Long $Y-X$, Yiu S-M. Predicting binary, discrete and continued IncRNA-disease associations via a unified framework based on graph regression. BMC Med Genet. 2017;10(Suppl 4):S5.

26. Bao W-Z, Jiang Z-C, Huang D-S. Novel human microbe-disease association prediction using network consistency projection. BMC Bioinformatics. 2017; 18(Suppl 16):S15.
27. Weifeng G, Shaowu Z, Qianqian S, Chengming Z, Tao Z, Luonan C. A novel algorithm for finding optimal driver nodes to target control complex networks and its applications for drug targets identification. BMC Genomics. 2017:18(Suppl 11):S6.

28. Lee K, Lee M, Kim D. Utilizing random Forest QSAR models with optimized parameters for target identification and its application to target-fishing server. BMC Bioinformatics. 2017;18(Suppl 16):S7.

29. Asami S, Kiga D, Konagaya A. Constraint-based perturbation analysis with cluster Newton method: a case study of personalized parameter estimations with irinotecan whole-body physiologically based pharmacokinetic model. BMC Systems Biol. 2017;11(Suppl 7):S13.

30. Ji Z, Wang B, Yan K, Dong L, Meng G, Shi L. A linear programming computational framework integrates phosphor-proteomics and prior knowledge to predict drug efficacy. BMC Systems Biol. 2017;11(Suppl 7):S1.

31. Pathak N, Lai M-L, Chen W-Y, Hsieh B-W, Yu G-Y, Yang J-M. Pharmacophore anchor models of flaviviral NS3 proteases lead to drug repurposing for DENV infection. BMC Bioinformatics. 2017;18(Suppl 16):S4.

32. Wang W, Yang X, Yang C, Guo XW, Zhang X, Wu C. Dependency-based long short term memory network for drug-drug interaction extraction. BMC Bioinformatics. 2017;18(Suppl 16):S9

33. Osato N. Characteristics of functional enrichment and gene expression level of human putative transcriptional target genes. BMC Genomics. 2017; 18(Suppl S11):S13.

34. Chen K, Liu L, Zhang X, Yuan Y, Ren S, Guo J, Wang Q, Liao P, Li S, Cui X, Li $Y-F$, Zheng Y. Phased secondary small interfering RNAs in Panax notoginseng. BMC Genomics. 2017;18(Suppl 11):S5.

35. Liu Y-C, Chiu Y-J, Li J-R, Sun C-H, Liu C-C, Huang H-D. Biclustering of transcriptome sequencing data reveals human tissue-specific circular RNAs. BMC Genomics. 2017;18(Suppl 11):S9.

36. Liany H, Rajapakse J, Karuturi RKM. MultiDCoX: multi-factor analysis of differential co-expression. BMC Bioinformatics. 2017;18(Suppl 16):S10.

37. Wang $X$, Lin $P$, Ho JWK. Discovery of cell-type specific DNA motif grammar in cis-regulatory elements using random Forest. BMC Genomics. 2017; 18(Suppl 11):S14.

38. Kumar S, Sharma A, Tsunoda T. An improved discriminative filter bank selection approach for motor imagery EEG signal classification using mutual information. BMC Bioinformatics. 2017;18(Suppl 16):S11.

39. Khushi M, Dean I, Teber E, Chircop M, Arthur J, Flores-Rodriguez N. Automated classification and characterization of the mitotic spindle following knockdown of a mitosis-related protein. BMC Bioinformatics. 2017; 18(Suppl 16):S13.

40. Zheng H, Wang R, Yu Z, Wang N, Gu Z, Zheng B. Automatic plankton image classification combining multiple view features via multiple kernel learning. BMC Bioinformatics. 2017;18(Suppl 16):S1.

41. Khan AM, Hu Y, Miotto O, Thevasagayam NM, Sukumaran R, HSA R, Brusic V, Tan TW, August JT. Analysis of viral diversity for vaccine target discovery. BMC Med Genet. 2017:10(Suppl 4):S1.

42. Lim WC, Khan AM. Mapping HLA-A2, A3 and B7 supertype-restricted T-cell epitopes in the ebolavirus proteome. BMC Genomics. 2017;18(Suppl 11):S2.

43. Pennisi M, Russo G, Ravalli S, Pappalardo F. Combining agent based-models and virtual screening techniques to predict the best citrus-derived vaccine adjuvants against human papilloma virus. BMC Bioinformatics. 2017; 18(Suppl 16):S8

44. Cao Y, Cao R, Huang Y, Zhou H, Liu Y, Li X, Zhong W, Hao P. A comprehensive study on cellular RNA editing activity in response to infections with different subtypes of influenza a viruses. BMC Genomics. 2017;18(Suppl 11):S4

45. Cao Y, Huang Y, Xu K, Liu Y, Xu Y, Hao P, Zhong W, Li X. Differential responses of innate immunity triggered by different subtypes of influenza a viruses in human and avian hosts. BMC Med Genet. 2017;10(Suppl 4):S4.

46. Huang Y, Cao Y, Li J, Liu Y, Zhong W, Li X, Chen C, Hao P. A survey on cellular RNA editing activity in response to Candida albicans infections. BMC Genomics. 2017;18(Suppl 11):S3.

47. Miao J, Han N, Qiang Y, Zhang T, Li X, Zhang W. 16sPIP: a comprehensive analysis pipeline for rapid pathogen detection in clinical samples based on 16S metagenomic sequencing. BMC Bioinformatics. 2017;18(Suppl 16):S22.

48. Herath D, Tang S-L, Tandon K, Ackland D, Halgamuge S. CoMet: a workflow using contig coverage and composition for binning a metagenomic sample with high precision. BMC Bioinformatics. 2017;18(Suppl 16):S14.

49. Huang K-Y, Chang T-Z, Jhong J-H, Chi Y-H, Li W-C, Chan C-L, Lai KR, Lee T-Y. Identification of natural antimicrobial peptides from bacteria through 
metagenomic and metatranscriptomic analysis of high-throughput transcriptome data of Taiwanese oolong teas. BMC Systems Biol. 2017; 11(Suppl 7):S3.

50. Wu Y-W. ezTree: an automated pipeline for identifying phylogenetic marker genes and inferring evolutionary relationships among uncultivated prokaryotic draft genomes. BMC Genomics. 2017;18(Suppl 11):S1.

51. Xing Y, Li G, Wang Z, Feng B, Song Z, Wu C. GTZ: a fast compression and cloud transmission tool optimized for FASTQ files. BMC Bioinformatics. 2017; 18(Suppl 16):S20.

52. Low J, Khang TF, Tammi M. CORNAS: coverage-dependent RNA-Seq analysis of gene expression data without biological replicates. BMC Bioinformatics. 2017;18(Suppl 16):S21.

53. Wang Y, Li G, Ma M, He F, Song Z, Zhang W, Wu C. GT-WGS: an efficient and economic tool for large-scale WGS analyses based on the AWS cloud service. BMC Genomics. 2017;18(Suppl 11):S8.

54. Zhao Y, Sun C, Zhao D, Zhang Y, You Y, Jia X, Yang J, Wang L, Wang J, Fu $H$, Kang $Y$, Chen F, Jun $Y$, WU J, Xiao J. PGAP-X: extension on pan-genome analysis pipeline. BMC Genomics. 2017;18(Suppl 11):S11.

55. Makita Y, Kawashima M, Lau NS, Matsui M, Othman AS. Construction of Pará rubber tree genome and multi-transcriptome database accelerates rubber researches. BMC Genomics. 2017;18(Suppl 11):S7.

56. Cheng L, Jiang Y, Ju H, Sun J, Zhou M, Hu Y, Peng J. InfAcrOnt: calculating cross-ontology term similarities using information flow by a random walk. BMC Genomics. 2017;18(Suppl 11):S12

57. Peng J, Wang $H$, Lu J, Hui W, Wang $Y$, Shang $X$. Identifying term relations cross different gene ontology categories. BMC Bioinformatics. 2017;18(Suppl 16):S6.

58. Liu Q, Wang J, Zhu Y, He Y. Ontology-based systematic representation and analysis of traditional Chinese drugs against rheumatism. BMC Systems Biol. 2017;11(Suppl 7):S10.

59. Kao H-J, Weng S-L, Huang K-Y, Kaunang F-J, Hsu JB-K, Huang C-H, Lee T-Y. MDD-Carb: a combinatorial model for the identification of protein carbonylation sites with substrate motifs. BMC Systems Biol. 2017; 11(Suppl 7):S11.

60. López Y, Sharma A, Dehzangi A, Lal SP, Taherzadeh G, Sattar A, Tsunoda T. Success: evolutionary and structural properties of amino acids prove effective for succinylation site prediction. BMC Genomics. 2017;18(Suppl 11):S10.

61. Su M-G, Weng JT-Y, Hsu JB-K, Huang K-H, Chi Y-H, Lee TY. Investigation and identification of functional post-translational modification sites associated with drug binding and protein-protein interactions. BMC Systems Biol. 2017; 11(Suppl 7):S6

62. Hardianto A, Yusuf M, Liu F, Ranganathan S. Exploration of charge states of balanol analogues acting as ATP-competitive inhibitors in kinases. BMC Bioinformatics. 2017;18(Suppl 16):S2.

63. Deng L, Fan C, Zeng Z. A sparse autoencoder-based deep neural network for protein solvent accessibility and contact number prediction. BMC Bioinformatics. 2017:18(Suppl 16):S18.

64. Yen C-Y, Lin J-C, Chen K-T, Lu CL. R3D-BLAST2: an improved search tool for similar RNA 3D substructures. BMC Bioinformatics. 2017;18(Suppl 16):S3.

\section{Submit your next manuscript to BioMed Central and we will help you at every step:}

- We accept pre-submission inquiries

- Our selector tool helps you to find the most relevant journal

- We provide round the clock customer support

- Convenient online submission

- Thorough peer review

- Inclusion in PubMed and all major indexing services

- Maximum visibility for your research

Submit your manuscript at www.biomedcentral.com/submit

) Biomed Central 